\title{
ASYMPTOTIC DISSIPATIVITY OF THE DIFFUSION PROCESS IN THE ASYMPTOTIC SMALL DIFFUSION SCHEME
}

\author{
Anastasiia Kinash $^{1}$, Yaroslav Chabanyuk ${ }^{2}$, Uliana Khimka ${ }^{1}$ \\ ${ }^{l}$ National University Lviv Polytechnic, Lviv, Ukraine \\ ${ }^{2}$ Lublin University of Technology, Lublin, Poland \\ anastasiiakinash@gmail.com,yaroslav_chab@yahoo.com,ulyana.himka@gmail.com
}

\begin{abstract}
This paper considers the random evolution with Markov switching. The resulting limited process is diffusion and depends on the small series parameter. The sufficient conditions of dissipativity of the limited process were obtained. Since the conditions of the Model Limit theorem and dissipativity conditions were set asymptotic dissipativity of the output process.
\end{abstract}

Keywords: small series parameter, diffusion, asymptotic dissipativity

\section{Introduction}

One of the main problems in the theory of random processes is the establishment of sufficient conditions for convergence to diffusion processes [1]. Random evolution of continuous and discrete types is considered in [2]. In particular, the solution of the singular perturbation problem is obtained for random processes with Markov and semi-Markov switching. Markov and semi-Markov processes are considered in the paper [3] for queuing systems with a single server and maintenance time, depending on the length of the queue. Work [4] deals with a stochastic process with Markov switching in the scheme of asymptotically small diffusion.

On the other hand, dissipativity as a property of the process is widely discussed in the literature. The book [5] deals with dissipativity as deterministic as well as random systems. Specifically, dissipativity of random systems with diffuse component is investigated in [6]. The important point to establish the system dissipativity conditions is the use of the Lyapunov function. In [7] the conditions of dissipativity of impulsive systems are studied in terms of Lyapunov function defined for this system. Asymptotic dissipativity of random evolution was previously received with diffusion [8] and impulse perturbation in the diffusion approximation scheme.

In this paper the stochastic process is considered with diffusion perturbation in the asymptotically small diffusion scheme. Asymptotic dissipativity derives from dissipativity of the limited evolution and conditions of the Model Limit theorem. 


\section{Problem formulation}

Considering the stochastic system with diffusion perturbation which is defined by stochastic differential equation [2]

$$
\begin{gathered}
d u^{\varepsilon}(t)=C\left(u^{\varepsilon}(t) ; x\left(\frac{t}{\varepsilon^{3}}\right)\right) d t+\varepsilon^{-1} C_{0}\left(u^{\varepsilon}(t) ; x\left(\frac{t}{\varepsilon^{3}}\right)\right) d t+ \\
+\varepsilon^{1 / 2} \sigma\left(u^{\varepsilon}(t) ; x\left(\frac{t}{\varepsilon^{3}}\right)\right) d w(t),
\end{gathered}
$$

where $u^{\varepsilon}(t), t \geq 0$ - random evolution, $u \in R^{d} ; C_{0}(u ; x)$ - singular perturbation of the regression function $C(u ; x) ; w(t)$ - Wiener process; $\sigma(u, x)$ - diffusion.

Markov process $x(t)>0$ is defined in the dimensional space phase of states $(X, \mathbf{X})$ with stationary distribution $\pi(B), B \in \mathbf{X}$.

The generator of the Markov process $x(t)$ is given by ratio

$$
\boldsymbol{Q} \phi(x)=q(x) \int_{X} Q(x, d y)[\phi(y)-\phi(x)] .
$$

For generator $\boldsymbol{Q}$ potential $\boldsymbol{R}_{0}$ is determined, which has the form $\boldsymbol{R}_{0}=\Pi-(\Pi+\boldsymbol{Q})^{-1}$, where $\Pi \phi(x)=\int_{X} \pi(d x) \phi(x)$ - projector on the subspace of zeroes of operator $\boldsymbol{Q}: N_{\boldsymbol{Q}}=\{\phi: \boldsymbol{Q} \phi=0\}$.

Limited evolution of system (1) has representation

$$
d u(t)=C(u) d t+\frac{1}{2} \varepsilon B(u) d w(t)
$$

where

$$
C(u)=\int_{X} C(u ; x) \pi(d x)
$$

Limited diffusion $\sigma$ is determined from the ratio $\sigma(u) \sigma^{*}(u)=B(u)$, where

$$
B(u)=\int_{X} \sigma^{2}(u ; x) \pi(d x)
$$

Let the condition of balance hold

$$
\int_{X} \pi(d x) C_{0}(u ; x) \equiv 0 .
$$


Operator $\tilde{\boldsymbol{L}}(x)$ takes the form

$$
\tilde{\boldsymbol{L}}(x)=[C(u)-C(u, x)] \phi^{\prime}(u)+\left[B(u)-\frac{1}{2} \varepsilon \sigma^{2}(u, x)\right] \phi^{\prime \prime}(u) .
$$

\section{Main result}

Theorem. Let the Lyapunov function $V(u) \in C^{4}\left(R^{d}\right)$ of system

$$
\frac{d u}{d t}=C(u)
$$

satisfies the conditions

$$
\begin{aligned}
& C 1:\left|C_{0}(u, x) \boldsymbol{R}_{0}\left[C_{0}(u, x) V^{\prime}(u)\right]^{\prime}\right|<M_{1} V(u), M_{1}>0 ; \\
& C 2:\left|A_{1}(u, x) \boldsymbol{R}_{0}\left[\tilde{L}(u, x) V^{\prime}(u)\right]^{\prime}\right|<M_{2} V(u), M_{2}>0 ; \\
& C 3:\left|C_{0}(u, x) \boldsymbol{R}_{0}\left[C_{0}(u, x) V^{\prime}(u)\right]^{\prime}\right|<M_{3} V(u), M_{3}>0 ; \\
& C 4:\left|A_{1}(u, x) \boldsymbol{R}_{0}\left[\tilde{L}(u, x) V^{\prime}(u)\right]^{\prime}\right|<M_{4} V(u), M_{4}>0 .
\end{aligned}
$$

Under the condition of balance (6) and inequalities

$$
\begin{gathered}
C(u) V^{\prime}(u)<-c_{1} V(u), c_{1}>0, \\
\sup _{u \in R^{d}}\|\sigma(u)\|<c_{2}, c_{2}>0,
\end{gathered}
$$

system (1) is asymptotic dissipative [2].

Proof of the theorem:

Lemma 1. Generator of two-component Markov process

$$
u^{\varepsilon}(t), x_{t}^{\varepsilon}=x\left(\frac{t}{\varepsilon^{3}}\right), t \geq 0
$$

in Banach space $\mathbf{B}\left(R^{d}, X\right)$ of real functions $\phi(u, x) \in C^{2,0}\left(R^{d}, X\right)$ has form

$$
\boldsymbol{L}^{\varepsilon} \phi(u, x)=\varepsilon^{-3} \boldsymbol{Q} \phi(u, x)+\varepsilon^{-1} \boldsymbol{C}_{0}(x) \phi(u, x)+\boldsymbol{A}_{1}(x) \phi(u, x),
$$

where

$$
A_{1}(x) \phi(u, x)=C(u, x) \phi^{\prime}(u, x)+\frac{1}{2} \varepsilon \sigma^{2}(u, x) \phi^{\prime \prime}(u, x)
$$

and

$$
C_{0}(x) \phi(u)=C_{0}(u, x) \phi^{\prime}(u, x)
$$


Proof: The generator of two-component Markov process (10) on the test-function $\phi(u, x)$ is given by

$$
\boldsymbol{L}^{\varepsilon} \phi(u, x)=\lim _{\Delta \rightarrow 0} \frac{1}{\Delta}\left[E\left[\phi\left(u^{\varepsilon}(t+\Delta), x_{t+\Delta}^{\varepsilon}\right) \mid u^{\varepsilon}(t)=u, x_{t}^{\varepsilon}=x\right]-\phi(u, x)\right] .
$$

Let us compute the conditional expectation

$$
E\left[\phi\left(u^{\varepsilon}(t+\Delta), x_{t+\Delta}^{\varepsilon}\right) \mid u^{\varepsilon}(t)=u, x_{t}^{\varepsilon}=x\right]=E_{u, x} \phi\left(u^{\varepsilon}+\Delta u^{\varepsilon}, x_{t+\Delta}^{\varepsilon}\right) .
$$

The indicators of system time spent in the state $x$ are determined from the ratios

$$
\begin{aligned}
& I\left(\theta_{t}>\varepsilon^{-3} \Delta\right)=e^{-\varepsilon^{-3} q(x) \Delta}=1-\varepsilon^{-3} q(x) \Delta+o(\Delta), \\
& I\left(\theta_{t} \leq \varepsilon^{-3} \Delta\right)=1-e^{-\varepsilon^{-3} q(x) \Delta}=\varepsilon^{-3} q(x) \Delta+o(\Delta),
\end{aligned}
$$

since the distribution function of the time spent in a state $x$ has an exponential distribution.

Hence,

$$
\begin{gathered}
E_{u, x} \phi\left(u^{\varepsilon}+\Delta u^{\varepsilon}, x_{t+\Delta}^{\varepsilon}\right)=E_{u, x}\left[\phi\left(u+\Delta u^{\varepsilon}, x_{t+\Delta}^{\varepsilon}\right)\right]\left[\left(\theta_{t}>\varepsilon^{-3} \Delta\right)+I\left(\theta_{t} \leq \varepsilon^{-3} \Delta\right)\right]= \\
=E_{u, x}\left[\phi\left(u+\Delta u^{\varepsilon}, x\right)\left(\theta_{t}>\varepsilon^{-3} \Delta\right)\right]+E_{u, x}\left[\phi\left(u+\Delta u^{\varepsilon}, x_{t+\Delta}^{\varepsilon}\right) I\left(\theta_{t} \leq \varepsilon^{-3} \Delta\right)\right]= \\
=E_{u, x}\left[\phi\left(u+\Delta u^{\varepsilon}, x\right)\left(1-\varepsilon^{-3} q(x) \Delta+o(\Delta)\right)\right]+ \\
+E_{u, x}\left[\phi\left(u+\Delta u^{\varepsilon}, x_{t+\Delta}^{\varepsilon}\right)\left(\varepsilon^{-3} q(x) \Delta+o(\Delta)\right)\right]= \\
=E_{u, x}\left[\phi\left(u+\Delta u^{\varepsilon}, x\right)\right]-\varepsilon^{-3} q(x) E_{u, x}\left[\phi\left(u+\Delta u^{\varepsilon}, x\right)\right] \Delta+ \\
+\varepsilon^{-3} q(x) E_{u, x}\left[\phi\left(u+\Delta u^{\varepsilon}, x_{t+\Delta}^{\varepsilon}\right)\right] \Delta+o(\Delta) .
\end{gathered}
$$

Consider the second term separately. From decomposition to Taylor series obtain

$$
\varepsilon^{-3} q(x) E_{u, x}\left[\phi\left(u+\Delta u^{\varepsilon}, x\right)\right] \Delta=\varepsilon^{-3} q(x)\left[E_{u, x} \phi(u, x)+E_{u, x}\left[\phi^{\prime}(u, x) \Delta u\right]\right] \Delta+o(\Delta) .
$$

From here

$$
\begin{aligned}
E_{u, x} \phi\left(u^{\varepsilon}\right. & \left.+\Delta u^{\varepsilon}, x_{t+\Delta}^{\varepsilon}\right)=E_{u, x}\left[\phi\left(u+\Delta u^{\varepsilon}, x\right)\right]+\varepsilon^{-3} q(x) E_{u, x}\left[\phi\left(u+\Delta u^{\varepsilon}, x_{t+\Delta}^{\varepsilon}\right)\right] \Delta- \\
& -\varepsilon^{-3} q(x)\left[E_{u, x} \phi(u, x)\right]-\varepsilon^{-3} q(x)\left[E_{u, x}\left[\phi^{\prime}(u, x) \Delta u\right]\right] \Delta+o(\Delta) .
\end{aligned}
$$


By the substitution received results in (13)

$$
\begin{gathered}
\boldsymbol{L}^{\varepsilon} \phi(u, x)=\lim _{\Delta \rightarrow 0} \frac{1}{\Delta} E_{u, x}\left[\phi\left(u+\Delta u^{\varepsilon}, x\right)\right]+\varepsilon^{-3} q(x) \lim _{\Delta \rightarrow 0} \frac{1}{\Delta} E_{u, x}\left[\phi\left(u+\Delta u^{\varepsilon}, x_{t+\Delta}^{\varepsilon}\right)\right] \Delta- \\
-\varepsilon^{-3} q(x) \lim _{\Delta \rightarrow 0} \frac{1}{\Delta}\left[E_{u, x} \phi(u, x)\right] \Delta-\varepsilon^{-3} q(x) \lim _{\Delta \rightarrow 0} \frac{1}{\Delta}\left[E_{u, x}\left[\phi^{\prime}(u, x) \Delta u\right]\right] \Delta- \\
-\phi(u, x)+o(\Delta)= \\
=\lim _{\Delta \rightarrow 0} \frac{1}{\Delta} E_{u, x}\left[\phi\left(u+\Delta u^{\varepsilon}, x\right)\right]+\varepsilon^{-3} q(x) \lim _{\Delta \rightarrow 0} E_{u, x}\left[\phi\left(u+\Delta u^{\varepsilon}, x_{t+\Delta}^{\varepsilon}\right)\right]- \\
-\varepsilon^{-3} q(x) \lim _{\Delta \rightarrow 0}\left[E_{u, x} \phi(u, x)\right]-\varepsilon^{-3} q(x) \lim _{\Delta \rightarrow 0}\left[E_{u, x}\left[\phi^{\prime}(u, x) \Delta u\right]\right]-\phi(u, x)+o(\Delta) .
\end{gathered}
$$

From the Taylor series

$$
\begin{gathered}
\varepsilon^{-3} q(x) E_{u, x}\left[\phi\left(u+\Delta u^{\varepsilon}, x_{t+\Delta}^{\varepsilon}\right)\right]=\varepsilon^{-3} q(x) E_{u, x}\left[\phi\left(u, x_{t+\Delta}\right)\right]+ \\
+\varepsilon^{-3} q(x) E_{u, x}\left[\phi^{\prime}\left(u, x_{t+\Delta}\right) \Delta u\right]+o(\Delta) .
\end{gathered}
$$

Therefore,

$$
\begin{gathered}
\boldsymbol{L}^{\varepsilon} \phi(u, x)=\lim _{\Delta \rightarrow 0} \frac{1}{\Delta} E_{u, x}\left[\phi\left(u+\Delta u^{\varepsilon}, x\right)\right]-\varepsilon^{-3} q(x) \lim _{\Delta \rightarrow 0}\left[E_{u, x} \phi(u, x)\right]- \\
-\varepsilon^{-3} q(x) \lim _{\Delta \rightarrow 0} E_{u, x}\left[\phi^{\prime}(u, x) \Delta u\right]+\varepsilon^{-3} q(x) \lim _{\Delta \rightarrow 0} E_{u, x}\left[\phi\left(u, x_{t+\Delta}\right)\right]+ \\
+\varepsilon^{-3} q(x) \lim _{\Delta \rightarrow 0} E_{u, x}\left[\phi^{\prime}\left(u, x_{t+\Delta}\right) \Delta u\right]-\phi(u, x)+o(\Delta) .
\end{gathered}
$$

Taking into account the representation of Markov process generator (2)

$$
q(x) \lim _{\Delta \rightarrow 0}\left[E_{u, x}\left[\phi\left(u, x_{t+\Delta}\right)\right]-E_{u, x}[\phi(u, x)]\right]=\lim _{\Delta \rightarrow 0} \boldsymbol{Q} \phi(u, x)=\boldsymbol{Q} \phi(u, x) .
$$

Consequently,

$$
\begin{aligned}
& \boldsymbol{L}^{\varepsilon} \phi(u, x)= \lim _{\Delta \rightarrow 0} \frac{1}{\Delta} E_{u, x}\left[\phi\left(u+\Delta u^{\varepsilon}, x\right)\right]+\varepsilon^{-3} \boldsymbol{Q} \phi(u, x)- \\
& \varepsilon^{-3} q(x) \lim _{\Delta \rightarrow 0} E_{u, x}\left[\phi^{\prime}(u, x) \Delta u\right]+ \\
&+\varepsilon^{-3} q(x) \lim _{\Delta \rightarrow 0} E_{u, x}\left[\phi^{\prime}\left(u, x_{t+\Delta}\right) \Delta u\right]-\phi(u, x)+o(\Delta) .
\end{aligned}
$$

From system (1) get

$$
\begin{gathered}
u_{t+\Delta}=u_{t}+\int_{t}^{t+\Delta} C\left(u^{\varepsilon}(s) ; x\left(\frac{s}{\varepsilon^{3}}\right)\right) d s+\varepsilon^{-1} \int_{t}^{t+\Delta} C_{0}\left(u^{\varepsilon}(s) ; x\left(\frac{s}{\varepsilon^{3}}\right)\right) d s+ \\
+\varepsilon^{1 / 2} \int_{t}^{t+\Delta} \sigma\left(u^{\varepsilon}(s) ; x\left(\frac{s}{\varepsilon^{3}}\right)\right) d w(s) .
\end{gathered}
$$


Thus,

$$
\begin{aligned}
u_{t+\Delta}-u_{t}=\int_{t}^{t+\Delta} C( & \left.u^{\varepsilon}(s) ; x\left(\frac{s}{\varepsilon^{3}}\right)\right) d s+\varepsilon^{-1} \int_{t}^{t+\Delta} C_{0}\left(u^{\varepsilon}(s) ; x\left(\frac{s}{\varepsilon^{3}}\right)\right) d s+ \\
& +\varepsilon^{1 / 2} \int_{t}^{t+\Delta} \sigma\left(u^{\varepsilon}(s) ; x\left(\frac{s}{\varepsilon^{3}}\right)\right) d w(s) .
\end{aligned}
$$

Substituting received result to second limit of expression for the generator $L^{\varepsilon} \phi(u, x)$

$$
\begin{gathered}
\lim _{\Delta \rightarrow 0}\left[E_{u, x}\left[\phi^{\prime}\left(u, x_{t+\Delta}\right)-\phi^{\prime}(u, x)\right] \Delta u\right]= \\
=\lim _{\Delta \rightarrow 0} E_{u, x}\left[\phi^{\prime}\left(u, x_{t+\Delta}\right)-\phi^{\prime}(u, x)\right] \int_{t}^{t+\Delta} C\left(u^{\varepsilon}(s) ; x\left(\frac{s}{\varepsilon^{3}}\right)\right) d s+ \\
+\varepsilon^{-1} \lim _{\Delta \rightarrow 0} E_{u, x}\left[\phi^{\prime}\left(u, x_{t+\Delta}\right)-\phi^{\prime}(u, x)\right] \int_{t}^{t+\Delta} C_{0}\left(u^{\varepsilon}(s) ; x\left(\frac{s}{\varepsilon^{3}}\right)\right) d s+ \\
+\varepsilon^{1 / 2} \lim _{\Delta \rightarrow 0} E_{u, x}\left[\phi^{\prime}\left(u, x_{t+\Delta}\right)-\phi^{\prime}(u, x)\right] \int_{t}^{t+\Delta} \sigma\left(u^{\varepsilon}(s) ; x\left(\frac{s}{\varepsilon^{3}}\right)\right) d w(s)=0 .
\end{gathered}
$$

Considering the obtained results

$$
\boldsymbol{L}^{\varepsilon} \phi(u, x)=\lim _{\Delta \rightarrow 0} \frac{1}{\Delta} E_{u, x}\left[\phi\left(u+\Delta u^{\varepsilon}, x\right)\right]+\varepsilon^{-3} \boldsymbol{Q} \phi(u, x)-\phi(u, x)+o(\Delta) .
$$

Replace

$$
z=u+\int_{t}^{t+\Delta} C\left(u^{\varepsilon}(s) ; x\left(\frac{s}{\varepsilon^{3}}\right)\right) d s+\varepsilon^{-1} \int_{t}^{t+\Delta} C_{0}\left(u^{\varepsilon}(s) ; x\left(\frac{s}{\varepsilon^{3}}\right)\right) d s
$$

So,

$$
\begin{gathered}
\lim _{\Delta \rightarrow 0} \frac{1}{\Delta} E_{u, x}\left[\phi\left(u+\Delta u^{\varepsilon}, x\right)\right]=\lim _{\Delta \rightarrow 0} \frac{1}{\Delta} E_{u, x}\left[\phi\left(z+\int_{t}^{t+\Delta} \sigma\left(u^{\varepsilon}(s) ; x\left(\frac{s}{\varepsilon^{3}}\right)\right) d w(s), x\right)\right]= \\
=\lim _{\Delta \rightarrow 0} \frac{1}{\Delta} E_{u, x}\left[\phi\left(z+\int_{t}^{t+\Delta} \sigma\left(u^{\varepsilon}(s) ; x\left(\frac{s}{\varepsilon^{3}}\right)\right) d w(s)-\phi(z, x)+\phi(z, x), x\right)\right]= \\
=\lim _{\Delta \rightarrow 0} \frac{1}{\Delta} E_{u, x}\left[\phi\left(z+\int_{t}^{t+\Delta} \sigma\left(u^{\varepsilon}(s) ; x\left(\frac{s}{\varepsilon^{3}}\right)\right) d w(s)-\phi(z, x), x\right)\right]+\lim _{\Delta \rightarrow 0} \frac{1}{\Delta} E_{u, x}[\phi(z, x)] .
\end{gathered}
$$


Applying the Taylor series to the first term

$$
\begin{gathered}
E_{u, x}\left[\phi\left(z+\varepsilon^{1 / 2} \int_{t}^{t+\Delta} \sigma\left(u^{\varepsilon}(s) ; x\left(\frac{s}{\varepsilon^{3}}\right)\right) d w(s), x\right)\right]= \\
=E_{u, x}[\phi(z, x)]+\varepsilon^{1 / 2} E_{u, x}\left[\phi^{\prime}(z, x)\right] E_{u, x}\left[\int_{t}^{t+\Delta} \sigma\left(u^{\varepsilon}(s) ; x\left(\frac{s}{\varepsilon^{3}}\right)\right) d w(s)\right]+ \\
+\frac{1}{2} E_{u, x}\left[\phi^{\prime \prime}(z, x)\right] E_{u, x}\left[\int_{t}^{t+\Delta} \sigma\left(u^{\varepsilon}(s) ; x\left(\frac{s}{\varepsilon^{3}}\right)\right) d w(s)\right]^{2}+o(\Delta) .
\end{gathered}
$$

Hence,

$$
\begin{gathered}
\lim _{\Delta \rightarrow 0} \frac{1}{\Delta} E_{u, x}[\phi(u+\Delta u, x)]= \\
=\frac{1}{2} \lim _{\Delta \rightarrow 0} \frac{1}{\Delta} \varepsilon E_{u, x}\left[\phi^{\prime \prime}(z, x)\right] E_{u, x}\left[\int_{t}^{t+\Delta} \sigma\left(u^{\varepsilon}(s) ; x\left(\frac{s}{\varepsilon^{3}}\right)\right) d w(s)\right]^{2}+ \\
+\lim _{\Delta \rightarrow 0} \frac{1}{\Delta} E_{u, x}[\phi(z, x)]+o(\Delta) .
\end{gathered}
$$

Thus generator takes the form

$$
\begin{gathered}
\boldsymbol{L}^{\varepsilon} \phi(u, x)=\frac{\varepsilon}{2} \lim _{\Delta \rightarrow 0} \frac{1}{\Delta} E_{u, x}\left[\phi ^ { \prime \prime } \left(u+\int_{t}^{t+\Delta} C\left(u^{\varepsilon}(s) ; x\left(\frac{s}{\varepsilon^{3}}\right)\right) d s+\right.\right. \\
\left.\left.+\frac{1}{\varepsilon} \int_{t}^{t+\Delta} C_{0}\left(u^{\varepsilon}(s) ; x\left(\frac{s}{\varepsilon^{3}}\right)\right) d s, x\right)\right] \times E_{u, x}\left[\int_{t}^{t+\Delta} \sigma\left(u^{\varepsilon}(s) ; x\left(\frac{s}{\varepsilon^{3}}\right)\right) d w(s)\right]^{2}+ \\
+E_{u, x}[\phi(z, x)]+\varepsilon^{-3} \boldsymbol{Q} \phi(u, x)-\phi(u, x)+o(\Delta)= \\
=\frac{1}{2} \varepsilon \sigma^{2}(u, x) \phi^{\prime \prime}(u, x)+\varepsilon^{-3} \boldsymbol{Q} \phi(u, x)+E_{u, x}[\phi(z, x)-\phi(u, x)]+o(\Delta)= \\
=\frac{1}{2} \varepsilon \sigma^{2}(u, x) \phi^{\prime \prime}(u, x)+\varepsilon^{-3} \boldsymbol{Q} \phi(u, x)+ \\
+E_{u, x}\left[\phi(u, x)+\left(C(u, x)+\varepsilon^{-1} C_{0}(u, x)\right) \phi^{\prime}(u, x)-\phi(u, x)\right]+o(\Delta)= \\
=\varepsilon^{-3} \boldsymbol{Q} \phi(u, x)+C(u, x) \phi^{\prime}(u, x)+\varepsilon^{-1} C_{0}(u, x) \phi^{\prime}(u, x)+\frac{1}{2} \varepsilon \sigma^{2}(u, x) \phi^{\prime \prime}(u, x) .
\end{gathered}
$$

Taking into account expression (12), generator (11) is obtained.

Lemma 2. Limited generator $\boldsymbol{L}$ on the perturbed test-function

$$
\phi^{\varepsilon}(u, x)=\phi(u)+\varepsilon^{2} \phi_{2}(u, x)+\varepsilon^{3} \phi_{3}(u, x), \phi(u) \in C^{4}\left(R^{d}\right)
$$


determined by the solution of the singular perturbation problem

$$
\boldsymbol{L}^{\varepsilon} \phi(u, x)=\varepsilon^{-1}\left[\boldsymbol{Q} \phi_{2}(u, x)+C_{0} \phi(u)\right]+\left[\boldsymbol{Q} \phi_{3}(u, x)+\boldsymbol{A}_{1}(x) \phi(u)\right]+\varepsilon \theta^{\varepsilon}(x),
$$

where residual term $\theta^{\varepsilon}(x)$ has representation

$$
\begin{gathered}
\theta^{\varepsilon}(x)=\boldsymbol{C}_{0}(x) \phi_{2}(u, x)+\varepsilon \boldsymbol{C}_{0}(x) \phi_{3}(u, x)+ \\
+\varepsilon A_{1}(x) \phi_{2}(u, x)+\varepsilon^{2} \boldsymbol{A}_{1}(x) \phi_{3}(u, x) .
\end{gathered}
$$

Proof: Generator (11) on the perturbed test-function (14) has the form

$$
\begin{gathered}
\boldsymbol{L}^{\varepsilon} \phi(u, x)=\varepsilon^{-3} \boldsymbol{Q} \phi(u)+\varepsilon^{-1} \boldsymbol{Q} \phi_{2}(u, x)+\boldsymbol{Q} \phi_{3}(u, x)+ \\
+\varepsilon^{-1} \boldsymbol{C}_{0} \phi(u)+\varepsilon \boldsymbol{C}_{0} \phi_{2}(u, x)+\varepsilon^{2} \boldsymbol{C}_{0} \phi_{3}(u, x)+ \\
+\boldsymbol{A}_{1} \phi(u)+\varepsilon^{2} \boldsymbol{A}_{1} \phi_{2}(u, x)+\varepsilon^{3} \boldsymbol{A}_{1} \phi_{3}(u, x)= \\
=\varepsilon^{-3} \boldsymbol{Q} \phi(u)+ \\
+\varepsilon^{-1}\left[\boldsymbol{Q} \phi_{2}(u, x)+\boldsymbol{C}_{0} \phi(u)\right]+ \\
+\left[\boldsymbol{Q} \phi_{3}(u, x)+\boldsymbol{A}_{1} \phi(u)\right]+ \\
+\varepsilon\left[\boldsymbol{C}_{0} \phi_{2}(u, x)+\varepsilon \boldsymbol{C}_{0} \phi_{3}(u, x)+\varepsilon \boldsymbol{A}_{1} \phi_{2}(u, x)+\varepsilon^{2} \boldsymbol{A}_{1} \phi_{3}(u, x)\right] .
\end{gathered}
$$

Since $\boldsymbol{Q} \in N_{\boldsymbol{Q}}$, then

$$
\boldsymbol{Q} \phi(u) \equiv 0
$$

Therefore,

$$
\begin{gathered}
\boldsymbol{L}^{\varepsilon} \phi(u, x)=\varepsilon^{-1}\left[\boldsymbol{Q} \phi_{2}(u, x)+\boldsymbol{C}_{0} \phi(u)\right]+\left[\boldsymbol{Q} \phi_{3}(u, x)+\boldsymbol{A}_{1}(x) \phi(u)\right]+ \\
\quad+\varepsilon\left[\boldsymbol{C}_{0} \phi_{2}(u, x)+\varepsilon \boldsymbol{C}_{0} \phi_{3}(u, x)+\varepsilon \boldsymbol{A}_{1} \phi_{2}(u, x)+\varepsilon^{2} \boldsymbol{A}_{1} \phi_{3}(u, x)\right] .
\end{gathered}
$$

Given the appearance of residual term (16) get generator (15).

Lemma 3. The solution of singular perturbation problem for generator $L^{\varepsilon}$ on perturbed Lyapunov function

$$
V^{\varepsilon}(u, x)=V(u)+\varepsilon^{2} V_{2}(u, x)+\varepsilon^{3} V_{3}(u, x), V(u) \in C^{4}\left(R^{d}\right)
$$

has a representation

$$
\boldsymbol{L}^{\varepsilon} V^{\varepsilon}(u, x)=\boldsymbol{L} V(u)+\varepsilon \theta_{1}^{\varepsilon}(x) V(u),
$$

where limited generator $\boldsymbol{L}$ has form

$$
\boldsymbol{L} V(u)=C(u) V^{\prime}(u)+\frac{1}{2} \varepsilon B(u) V^{\prime \prime}(u)
$$


and residual term $\theta_{1}^{\varepsilon}(x)$ is defined by the ratio

$$
\begin{gathered}
\theta_{1}^{\varepsilon}(x)=\boldsymbol{C}_{0}(x) \boldsymbol{R}_{0} \boldsymbol{C}_{0}(x)+\varepsilon \boldsymbol{C}_{0}(x) \boldsymbol{R}_{0} \tilde{\boldsymbol{L}}(x)+ \\
+\varepsilon \boldsymbol{A}_{1}(x) \boldsymbol{R}_{0} \boldsymbol{C}_{0}(x)+\varepsilon^{2} \boldsymbol{A}_{1}(x) \boldsymbol{R}_{0} \tilde{\boldsymbol{L}}(x) .
\end{gathered}
$$

Proof: The generator (15) on the perturbed Lyapunov function (17) has representation

$$
\boldsymbol{L}^{\varepsilon} V(u, x)=\varepsilon^{-1}\left[\boldsymbol{Q} V_{2}(u, x)+\boldsymbol{C}_{0} V(u)\right]+\left[\boldsymbol{Q} V_{3}(u, x)+\boldsymbol{A}_{1}(x) V(u)\right]+\varepsilon \theta^{\varepsilon}(x) .
$$

From the first condition of singular perturbation problem solvability

$$
\begin{gathered}
\boldsymbol{Q} V_{2}(u, x)+\boldsymbol{C}_{0} V(u)=0, \\
\boldsymbol{Q} V_{2}(u, x)=-\boldsymbol{C}_{0} V(u) .
\end{gathered}
$$

Applying the balance condition (6) gets

$$
V_{2}(u, x)=\boldsymbol{R}_{0} \boldsymbol{C}_{0} V(u) .
$$

Using the second condition of solvability

$$
\boldsymbol{Q} V_{3}(u, x)+\boldsymbol{A}_{1}(x) V(u)=\boldsymbol{L} V(u),
$$

here $A_{1}(x)$ is given by (12).

$$
\boldsymbol{Q} V_{3}(u, x)=-\boldsymbol{A}_{1}(x) V(u)+\boldsymbol{L} V(u),
$$

where

$$
\boldsymbol{L}=\Pi \boldsymbol{A}_{1}(x)
$$

Denote by $\tilde{\boldsymbol{L}}(x)$ difference

$$
\tilde{\boldsymbol{L}}(x)=\boldsymbol{L}-A_{1}(x)
$$

Then

$$
\begin{aligned}
& \boldsymbol{Q} V_{3}(u, x)=\tilde{\boldsymbol{L}}(x) V(u), \\
& V_{3}(u, x)=\boldsymbol{R}_{0} \tilde{\boldsymbol{L}}(x) V(u) .
\end{aligned}
$$

That is generator (22), which has representation

$$
\boldsymbol{L} V(u)=V^{\prime}(u) \int_{X} C(u ; x) \pi(d x)+\frac{1}{2} \varepsilon V^{\prime \prime}(u) \int_{X} \sigma^{2}(u ; x) \pi(d x)
$$


By the substitution ratios (21) and (23) in residual term (16)

$$
\theta_{1}^{\varepsilon}(x)=\boldsymbol{C}_{0}(x) \boldsymbol{R}_{0} \boldsymbol{C}_{0}(x)+\varepsilon \boldsymbol{C}_{0}(x) \boldsymbol{R}_{0} \tilde{\boldsymbol{L}}(x)+\varepsilon \boldsymbol{A}_{1}(x) \boldsymbol{R}_{0} \boldsymbol{C}_{0}(x)+\varepsilon^{2} \boldsymbol{A}_{1}(x) \boldsymbol{R}_{0} \tilde{\boldsymbol{L}}(x) .
$$

Thus, taking into account expressions (4) and (5), generator (18) is obtained with limited generator (19) and residual term (20).

From the conditions $C 1-C 4$ of the theorem boundary on the residual term is of the form (20)

$$
\left\|\theta_{u}^{\varepsilon}(x) \phi(u, w)\right\|<M V(u) .
$$

From Lemma 3 and expression (24) the conditions of the Model Limit theorem take place ([2], p. 64)

$$
\left(u^{\varepsilon}(t), \eta^{\varepsilon}(t)\right) \rightarrow(\hat{u}(t), \sigma W(t)), \varepsilon \rightarrow 0 .
$$

Lyapunov function $V(u)$ satisfies the Lipschitz condition

$$
\left|V\left(u_{2}\right)-V\left(u_{1}\right)\right|<K\left|u_{2}-u_{1}\right|
$$

where $K$ - const.

Then the relation below is performed

$$
\frac{d^{(1)} V(u)}{d u} \leq \frac{d V(u)}{d u}+K\|\sigma(u)\||d W(t)|
$$

where $\left(d^{(1)} V(u) / d u\right)$ and $(d V(u) / d u)$ - derivatives of the Lyapunov function calculated along the trajectory of the systems (3) and (7), respectively.

Applying expressions (8) and (9) ([5], p. 23), have

$$
\frac{d^{(1)} V(u)}{d u} \leq-c_{1} V(u)+K c_{2}|d W(t)| .
$$

Therefore, $V(u) \leq V\left(u_{0}\right) \exp \left\{\int_{0}^{t}-c_{1} d s\right\}+\int_{0}^{t} \exp \left\{\int_{s}^{t}-c_{1} d p\right\} K c_{2}|d W(s)| d s$.

Thus, $V(u) \leq V\left(u_{0}\right) \exp \left\{-c_{1} t\right\}+K c_{2} \int_{0}^{t} \exp \left\{-c_{1}(t-s)\right\}|d W(s)| d s$.

Finding the expectation of both parts inequality obtained

$$
E V(u) \leq V\left(u_{0}\right) \exp ^{-c_{1} t}+K c_{2} \int_{0}^{t} \exp ^{-c_{1}(t-s)} E|d W(s)| d s .
$$


Consequently, estimates are of the form ([5], p. 23)

$$
P\{|u(t)|>R\} \leq \frac{E V(u)}{\inf _{u \in R^{d}} V(u)}, R \rightarrow 0 .
$$

So, system (3) is dissipative. Herefrom and from the Modal Limit theorem follows that system (1) is asymptotic dissipative.

\section{Conclusions}

This result makes it possible to build a functional of action in the problem of large deviations with small diffusion for asymptotic dissipative stochastic evolutions. In the paper the asymptotic dissipativity of random evolution with Markov switching in the asymptotic small diffusion scheme is obtained. Balance condition on the singular perturbation of regression function is crucial for establishing convergence of the initial process to the limited evolution.

\section{References}

[1] Korolyuk V.S., Stability of stochastic systems in the diffusion approximation scheme, Ukr. Math. Jour. 1998, 50, 1, 36-47 (in Ukrainian).

[2] Korolyuk V.S., Limnios N., Stochastic Systems in Merging Phase Space, World Scientific Publishing 2005.

[3] Zhernovyi Yu., Kopytko B., Zhernovyi K., The busy period for the $M^{\theta} / \mathrm{G} / 1 / \mathrm{m}$ system with service time dependent of the queue length, Journal of Applied Mathematics and Computational Mechanics 2013, 12(4), 127-133.

[4] Kiikovska O.I., Chabanyuk Ya.M., Random evolution in the asymptotically small diffusion scheme with Markov switching, Cybernetics and Systems Analysis 2013, 49, 2, 164-169 (in Ukrainian).

[5] Khasminskii R.Z., Stability of Systems of Differential Equations with Random Perturbations of Their Parameters, Nauka, Moscow 1969, 368 (in Russian).

[6] Khasminskii R.Z., About dissipativity of random processes defined by differential equations, Probl. peredachu infor. 1965, 1, 1, 88-104 (in Russian).

[7] Samoilenko A.M., Stangytckyi O.M., Qualitative and asymptotic analysis of differential equations with random perturbations, Naukova dumka 2009, 336 (in Ukrainian).

[8] Kinash A.V., Chabanyuk Ya.M., Khimka U.T., Asymptotic dissipativity of diffusion process, Mathematical and Computer Modeling, Series: Physical and Mathematical Sciences 2014, 11, 78-87 (in Ukrainian). 